\title{
Managing and monitoring tuberculosis using web-based tools in combination with traditional approaches
}

This article was published in the following Dove Press journal:

Clinical Epidemiology

21 November 2013

Number of times this article has been viewed

\section{Ann LN Chapman' \\ Thomas C Darton ${ }^{2}$ \\ Rachel A Foster'}

'Department of Infection and Tropical Medicine, Sheffield Teaching Hospitals NHS Foundation Trust, Sheffield, ${ }^{2}$ Oxford Vaccine Group, Centre for Clinical Vaccinology and Tropical Medicine, University of Oxford, Oxford, UK
Correspondence: Ann LN Chapman Department of Infection and Tropical Medicine, Royal Hallamshire Hospital, Glossop Road, Sheffield, SIO 2JF, UK Tel +44 I I 42268874 Fax +44 II4 2268875

Email ann.chapman@sth.nhs.uk

\begin{abstract}
Tuberculosis (TB) remains a global health emergency. Ongoing challenges include the coordination of national and international control programs, high levels of drug resistance in many parts of the world, and availability of accurate and rapid diagnostic tests. The increasing availability and reliability of Internet access throughout both affluent and resource-limited countries brings new opportunities to improve TB management and control through the integration of web-based technologies with traditional approaches. In this review, we explore current and potential future use of web-based tools in the areas of TB diagnosis, treatment, epidemiology, service monitoring, and teaching and training.
\end{abstract}

Keywords: tuberculosis, information communication technology, Internet

\section{Introduction}

Tuberculosis (TB) continues to be a major global cause of death and morbidity, disproportionately affecting the poorest communities. In 2011, 8.7 million new cases and 1.4 million deaths attributable to TB were reported. ${ }^{1}$ Despite the availability of effective treatment regimes for the majority of cases, TB control remains poor in many countries due to antibiotic resistance, coinfection with human immunodeficiency virus (HIV), and, most significantly, deficiencies in coordinating national TB programs. In 1993, the World Health Organization (WHO) declared TB a global public health emergency and called on governments across the globe to prioritize TB control. Much progress has been made since then through the work of partnerships such as the Stop TB Campaign, which aims to halt the current epidemic by $2015 .^{2}$

Since the invention of the World Wide Web in the early 1990s, technologic advances have revolutionized daily life for people throughout the globe. Information communication technology is being increasingly used in health care settings, and has the potential to contribute significantly to health care provision, both as regards cost savings and quality of care. A WHO resolution on eHealth in 2005 encouraged member states to collaborate and provide mutual support for its integration into health systems to improve health care, surveillance, and education. Although there remains a "digital divide" with patchy provision of information communication technology access globally, rapid advances in mobile telephone and wireless technology, and its increasing availability in otherwise resource-limited settings, are improving this situation. ${ }^{3,4}$ Integrating control programs with web-enabled applications and wireless communication offers the potential for significant improvements in TB service provision, with major benefits for the global fight against TB. In this review, we describe 
the broad range of tools and applications available from the Internet or which incorporate real-time electronic data transfer, aimed at improving the management of TB. We focus on five key areas (Table 1):

- diagnosis

- treatment

- contact tracing and epidemiology

- service performance monitoring and quality assurance

- teaching and training.

We assess current processes and future opportunities, in comparison with those available for other infectious diseases, and discuss how these advancements are making real differences in clinical practice.

\section{Search strategy}

A search of the English language peer-reviewed literature between 2005 and 2013 was conducted using PubMed. Search terms included "tuberculosis AND Internet", "tuberculosis AND web", and "electronic patient record TB". Further articles were identified by review of the reference lists, and through targeted searches for literature on specific subcategories.

\section{Diagnosis of TB}

Diagnosis of active TB infection remains a challenge throughout the world due to limited facilities for culture and sensitivity testing. In many places, sputum microscopy is the

Table I Web-based tools for TB

\begin{tabular}{|c|c|}
\hline Diagnosis & $\begin{array}{l}\text { - Laboratory networks } \\
\text { - Teleradiology } \\
\text { - Laboratory information systems } \\
\text { - Web-linked portable diagnostic tools } \\
\text { - Clinical decision support systems }\end{array}$ \\
\hline Treatment & $\begin{array}{l}\text { - Remote consultations/clinical support } \\
\text { - Electronic patient records } \\
\text { - Electronic medication monitors } \\
\text { - Directly observed and video-observed } \\
\text { therapy } \\
\text { - Patient tracking systems }\end{array}$ \\
\hline $\begin{array}{l}\text { Contact tracing and } \\
\text { epidemiology }\end{array}$ & $\begin{array}{l}\text { - Web-based contact tracing } \\
\text { - Automatic identification of individuals } \\
\text { for screening/contact tracing } \\
\text { - Web-based strain typing registries } \\
\text { - Networked national surveillance systems }\end{array}$ \\
\hline $\begin{array}{l}\text { Service performance } \\
\text { monitoring and quality }\end{array}$ & $\begin{array}{l}\text { - Development and monitoring of national } \\
\text { TB programs }\end{array}$ \\
\hline assurance & $\begin{array}{l}\text { - Data storage for quality assurance and } \\
\text { governance }\end{array}$ \\
\hline Teaching and training & $\begin{array}{l}\text { - Information resources } \\
\text { - Online training }\end{array}$ \\
\hline
\end{tabular}

Abbreviation: TB, tuberculosis. only diagnostic test available, and treatment is frequently started on the basis of radiologic changes or clinical features alone. Recent developments in portable polymerase chain reaction-based technology could allow more rapid confirmation of the diagnosis of TB, also providing early information about drug susceptibility, ${ }^{5,6}$ but may be unaffordable in resource-poor areas.

There is an increasing focus on improving access to existing and novel TB diagnostics as an important strategy in global TB control. The EXPANDx-TB program was launched by the WHO Global Laboratory Initiative, the Foundation for Innovative New Diagnostics, and the WHO Global Drug Facility in 2009. Funded by the UNITAID partnership, its aim is to speed up the diagnosis of TB in high-burden countries by increasing access to new diagnostic technologies. Two key components of the Stop TB strategy are to ensure early case detection and diagnosis through quality-assured bacteriology and to upgrade laboratory networks. ${ }^{2}$ Use of web-based systems in conjunction with traditional diagnostic processes will be essential to support progress towards these goals. However, this will require a comprehensive electronic infrastructure in order for real benefits to be achieved. The large amounts of initial capital investment and ongoing technologic support required for setting up such electronic systems present a huge barrier in resource-limited settings and will require ongoing international commitment to tackling the problem.

There are some excellent examples of interventions to improve TB diagnostic services in the developing world, such as those in Zanzibar, Tanzania, which include improvement of laboratory facilities and expert telemedicine support. ${ }^{7}$ This intervention involved overseas financial support, the use of WHO benchmarks for diagnostic quality, and political commitment to training and service improvement. A web-based link between regional and district laboratory reporting systems also increased the speed of reporting of results in Peru. ${ }^{8}$ Early confirmation of diagnosis and initiation of treatment is beneficial both to the individual patient and also to overall control of TB locally and nationally through reduction of ongoing transmission. This is particularly important in the control of drug-resistant TB, ${ }^{9}$ and the Peruvian web-based system also incorporates drug susceptibility data, allowing more rapid transfer of this information to inform clinical care and public health measures.

\section{Teleradiology}

The lack of specialist radiologists in many resource-limited settings presents another barrier to timely diagnosis of TB, 
particularly in areas with high HIV prevalence and consequently large numbers with smear-negative disease and atypical radiologic findings. Using the World Wide Web to transfer digital images for a remote radiologic opinion, a process termed teleradiology, overcomes this problem and may reduce the time taken, and increase the accuracy of TB diagnoses. In a feasibility study in Malawi, high-quality digital photographs were taken of original radiographs on a light box and the image transmitted to a radiologist in the US via the Internet. ${ }^{10}$ Images were selected for inclusion on the basis of local uncertainty about the findings; in $23.5 \%$ of cases, there was a change in patient management as a result. These changes included additional diagnoses of clinically unsuspected pulmonary $\mathrm{TB}$, and also radiologic exclusion of infection, thereby avoiding unnecessary treatment. The degree of consensus over interpretation of radiographs by clinicians in Malawi and by the radiologist in the US improved over the course of the study, illustrating an additional educational benefit. Such systems could potentially contribute to improved quality of patient care, although the authors stressed the importance of clear criteria for referral, shared knowledge of the pattern of disease in the local area, having a local "link person" to coordinate X-ray transfer and receive results, and allowing time for local radiology staff to adapt to the change.

A teleradiology link between peripheral hospitals in KwaZulu-Natal, South Africa, and a Durban teaching hospital also showed benefit, with 20 of 200 radiologic reviews leading to diagnosis of TB which would have otherwise been missed within the first month. Technical problems with inadequate phone links were experienced, however, limiting the effectiveness of the intervention. ${ }^{11}$ Others have highlighted the medicolegal and governance risks associated with teleradiology; close adherence to available guidance, such as that produced by the Royal College of Radiologists in UK, is therefore important. ${ }^{12,13}$

\section{Laboratory information management systems}

Where diagnostic TB laboratories do exist in resource-limited settings, limitations in infrastructure often impact patient care. Dunbar et $\mathrm{al}^{14}$ described inadequacies in information flow between laboratory and clinical services in Cape Town, South Africa. When laboratory records were cross-checked with treatment registers, it was found that a high percentage of bacteriologically confirmed cases were not recorded in the treatment registers, resulting in untreated cases and preventable onward transmission. It was concluded that an integrated electronic results management system would improve the management of laboratory results.

Laboratory information management systems facilitate the recording and sharing of information regarding samples, results, quality indicators, and instruments. They can also be used to share regulatory and safety information as well as training materials, and a number of open access systems are now available. The ongoing training and maintenance costs are a challenge in resource-poor settings, but if they are linked to improved laboratory networks and prompt timely appropriate treatment in the field, they have the potential to save money. Much work has been done in Peru by Blaya, Fraser, and others to improve TB laboratory services and results management systems, with very clear benefits. They demonstrated an $87 \%$ reduction of reporting errors in health centers using the e-Chasqui electronic laboratory information system compared with those using a paper-based system through reductions in missing results. ${ }^{15}$ In addition, the use of personal digital assistants resulted in faster processing of sputum smear and TB culture results. ${ }^{16}$ However, their work illustrated that the improvements only extended as far as the geographic reach of the electronic hardware introduced, ie, health centers without direct access to the information system did not benefit, highlighting again the importance of investing in comprehensive information technology infrastructure. ${ }^{17}$

In the future, novel web-based technologies may allow the results of diagnostic tests carried out in the field to be integrated rapidly with laboratory information systems. One group described the use of mobile electronic devices both to perform serologic testing for HIV and to upload results automatically to a database in "the cloud", from where they were synchronized with an electronic health record for patients in Rwanda. ${ }^{18}$ This technology could potentially be extended to TB, for example, using portable diagnostic systems on sputum samples.

The development of laboratory information management systems locally and nationally is likely to provide wider benefits across a range of infections beyond TB, and will clearly be useful to link developments across clinical areas. In the context of TB, one key linkage in many parts of the world is with HIV care, and there are opportunities for cost savings if approaches are combined, for example, in simultaneous collection, transport, and testing of samples, and links to national monitoring systems. It is important to have a collaborative approach, which may require national integration between different donor organizations. 


\section{Diagnosis of latent TB}

Diagnosis and treatment of latent TB infection is an important control strategy in low incidence countries. Despite the development of interferon gamma release assays over the past decade, the tuberculin skin test remains a common diagnostic tool for latent TB. However, its sensitivity and specificity are limited, and there is a lack of consensus regarding its interpretation in different groups. Menzies et a ${ }^{19}$ developed a web-based algorithm incorporating medical and radiologic risk factors, tuberculin skin test reaction size, and prior vaccination with Bacille-Calmette-Guerin (BCG) to aid the interpretation of tuberculin skin test results. They concluded that the algorithm could allow more accurate targeting of therapy, resulting in fewer cases of reactivation and fewer patients unnecessarily receiving treatment. Another group developed a computerized clinical decision support tool coupled with web-based documentation for use in assessing individuals with a high risk of latent TB, and found that compliance with national screening guidelines improved from $8.9 \%$ to $25.2 \%$ with use of the tool. ${ }^{20}$

\section{Treatment of TB}

At its simplest level, web-based technology can contribute to care of patients with $\mathrm{TB}$, for example, through use of emails to speed up communication, obtain second opinions, and provide educational materials. ${ }^{21-23}$ Remote consultations can be undertaken using online communication systems such as Skype. More complex systems can be used to document and track patients' progress through the treatment process, monitor adherence, detect those at risk of failing follow-up, and improve speed of communication within and between institutions. At a national level, networked electronic patient records can link into national surveillance systems and also provide a valuable research resource. ${ }^{3}$

\section{Electronic patient records}

Electronic patient records (EPRs) have been introduced in many countries in recent years. They have the potential to improve many aspects of clinical practice, including prescribing, patient follow-up, chronic disease management, and adherence to clinical guidelines. However, there are also potential challenges, including underestimation of time commitment by physicians, the need for expensive ongoing technical support, and concerns about the maintenance of patient confidentiality. ${ }^{24}$

With increasing availability and reliability of Internet access globally, there is recognition that EPRs could also contribute significantly to improved patient care in resource-limited areas. Although there are practical problems with establishing EPR systems in such settings, many can be overcome simply, for example, through the use of solar batteries where power supplies are unreliable, or through limiting the number of computers networked to a web-based central database. Where computerized records are available, web-based systems have significant advantages over locally held patient databases, many of which rapidly become overwhelmed by large amounts of patient information and are user-unfriendly and unwieldy. Fraser et $\mathrm{a}^{25}$ reviewed a number of systems in use in Africa, and Central and South America, and described the benefits of such systems. These included:

- clear documentation of clinical notes

- reduced consultation and waiting times

- decision support for prescribing medications, including reminders, allergy warnings, and information about drug interactions

- alerts for abnormal laboratory results

- facilitation of data provision for national outcome monitoring.

Systems developed locally are often adaptable for use in other geographic areas. The eChasqui system, for example, was transferred successfully from Peru to the Philippines, ${ }^{26}$ and has subsequently been implemented in over 40 countries as the Open Medical Record System. ${ }^{27}$

EPRs range from simple systems for storing locally entered patient data to more complex systems with links to other data sources relating to the patient, for example, digital radiology images, and laboratory and pharmacy information systems. ${ }^{28}$ In addition, they may facilitate other aspects of patient care, for example, through incorporation of clinical decision support systems, ${ }^{29}$ providing alerts to clinicians on drug interactions, timing of laboratory investigations or clinic recalls, or patients defaulting from treatment, through linkage with phone or text patient appointment reminders, and through providing a system for disseminating public health practice guidance. ${ }^{30}$

\section{Directly observed therapy monitoring systems}

A key strategy in global TB control is the use of directly observed therapy to ensure that patients adhere to medication and complete prescribed treatment courses. In areas of high TB prevalence, however, universal directly observed therapy may be unsustainable due to limited health care staff and infrastructure capacity. The use of electronic medication monitors has been advocated as an adjunct to directly 
observed therapy. ${ }^{31}$ These devices record when medication is removed from the container, thus providing a record of adherence. The simplest devices include a built-in electronic display, but some are able to transmit data to a central point via a telephone or wireless connection, allowing remote monitoring. ${ }^{32}$ Their use could allow health care workers to target directly observed therapy and treatment counseling to patients who are failing to comply with therapy, and could therefore result in improved treatment adherence, more efficient use of staff time, and increased program cost-effectiveness.

Another recent development includes the use of webbased technology to support directly observed therapy through "video-observed therapy". ${ }^{33}$ This is increasingly being used in the US for selected patients, and has the advantages of reduced travel costs and time for staff, easy regular visual contact, and increased privacy for patients.

\section{Patient tracking systems}

Electronic tracking systems to monitor effective treatment and follow-up of patients identified with multidrug-resistant TB or HIV have been reviewed by Fraser et al. ${ }^{34}$ They concluded that committed community health care workers provided with personal digital assistants or smart phones would be able to trace and treat patients who are lost to follow-up or never initiated treatment, to ensure that treatment courses are completed. The web-based tracking and results system in Peru, also now shared with the Philippines and elsewhere, brings clear benefit in reducing delays and errors, and improving service efficiency, although it is not yet clear if such systems are cost-effective in the long term. ${ }^{35}$

\section{Research and development}

At a more basic level, web-based technologies have contributed to TB management through the enablement of very large international open access databases containing mycobacterial genomic sequencing and protein data. These allow new searches for novel protein targets for new TB therapies, resistance mutations, and vaccine candidates. ${ }^{36-40}$ New technology has (and continues to) contributed to the sharing of knowledge and research collaboration at a global level, facilitating rapid advances in TB therapeutics.

\section{Contact tracing and epidemiology}

The identification and targeted testing of contacts of infectious cases of TB is an important TB control strategy. For the individual patient and their contacts, information communication technology can support traditional contact tracing activity through text and mobile phone communication with possible contacts. In the field of sexually transmitted infection, there are some reports of the use of Internet-based partner notification using email to initiate contact. ${ }^{41}$ Although the number of partners tested and treated was increased compared with standard methods, concerns were raised about the acceptability of this approach. ${ }^{42}$

EPRs have been successfully used to improve TB surveillance in Massachusetts, US, through identification of patients being started on TB therapy who had erroneously not been notified as new cases. A system was set up where the EPR automatically generated an alert in a predefined scenario, for example, when a patient was prescribed pyrazinamide, had samples sent for TB culture, or had a test result suggestive of active TB. This also improved contact tracing efforts by more accurately reporting active cases, leading to more comprehensive and more rapid identification of potential contacts. ${ }^{43}$ In Illinois, US, EPRs have been used to improve compliance with TB screening programs in patients commencing biologic therapies with tumor necrosis factor antagonists. ${ }^{44} \mathrm{All}$ patients with an electronic prescription for a tumor necrosis factor antagonist were flagged as requiring screening tests for TB and hepatitis B, as well as routine full blood count and liver function tests. With increasing use of immunosuppressive therapies for many inflammatory conditions, such an approach could be extended to a range of settings.

\section{Strain typing}

The development and refinement of strain typing methodologies offers the potential for improved contact tracing when used alongside traditional contact investigation activity. ${ }^{45}$ Mycobacterium tuberculosis isolates can be typed according to the number of repeat sequences at various sites or loci within the genome: the MIRU-VNTR (mycobacterial interspersed repetitive units-variable numbers of tandem repeats) method uses 24 loci. Each isolate is given a 24-digit code specifying the number of repeats at each locus; the codes are highly specific for that isolate and hence can be used to provide evidence for or against transmission from person to person. Strain typing data allow rapid identification of potential clusters of related cases, enabling early initiation of control measures ${ }^{46}$ The utility of strain typing is supported by the development of large web-based national and international strain registries which allow comparisons to be made between strains, for local contact tracing, and also for wider epidemiologic investigations, for example the MIRU-VNTRplus tool. ${ }^{47,48}$ Databases containing full genomic sequencing data can also be used as research tools to explore 
genotype-phenotype associations, for example, the open access $M$. tuberculosis genome divergence database. ${ }^{49}$

However, the increasing availability of Internet access worldwide can have unintended consequences: Ohkado et $\mathrm{al}^{50}$ described an outbreak of TB in Tokyo which was investigated using strain typing. They concluded that transmission was most likely to have occurred in Internet cafés!

\section{Surveillance}

National TB surveillance programs are required to obtain accurate information on TB incidence and management in order to plan national services and to contribute data to inform global TB epidemiology. In affluent countries such systems are usually web-based, allowing data from a number of reporting centers to be collated and rapid reports generated, for example, the surveillance system in operation in the US, which is linked to OTIS, an online interactive TB information system. Lew et al described the development of a new TB surveillance network and national control program across hundreds of health centers in Korea. ${ }^{51}$ The web-based format of the network allowed easy access at any time of day and by a wide range of health care workers. In the UK, surveillance data are collected via the enhanced TB surveillance system, established in 1999; again, reports are openly accessible online. Dominkovics et al described a useful application of a web-based national TB surveillance system in Barcelona, Spain. ${ }^{52}$ Residential addresses of TB patients notified to the central registry were geocoded and used to produce spatial density maps to assist management by health care workers in the field, and for use in public health decision-making.

Mor et $\mathrm{al}^{53}$ undertook a comparison of TB surveillance systems in 19 low-incidence industrialized countries. Seventeen of these used web-based systems, with only two countries (Denmark and Norway) submitting paper notifications. The authors speculated that improved webbased communication between national surveillance systems could improve global surveillance, as well as facilitating joint planning of TB services, contributing to improved coordination of immigrant health screening, and supporting joint research on TB in low-incidence countries.

Electronic TB surveillance systems are also advocated by WHO for resource-poor settings, and a series of recommendations has been developed. These were used in Botswana to develop an electronic TB register. ${ }^{54}$ Patient data were entered manually from reporting forms, and the software provided outputs including lists of treatment defaulters and reports of treatment outcomes and trends. One critical factor in the success of this project was the pre-existence of a robust and accurate paper-based system. Nadol et al examined three systems in operation in a range of settings in Africa, the Eastern Mediterranean region, East Asia, and Mexico to identify benefits and requirements of electronic surveillance. ${ }^{55}$ All involved direct entry of patient data from treatment cards. In two systems, data were stored on a central server, and in the third an online database was used, with access through both desktop computers and laptops or personal digital assistants, allowing generation of real-time reports. The authors discussed the advantages and disadvantages of electronic surveillance systems, and again pointed out the importance of a well established paper-based system, and also the cost of infrastructure and maintenance, as well as the need for training and technical support.

\section{Service performance monitoring and quality assurance}

In order for different nations with different resources and burdens of TB to make the best use of their resources, modeling tools are being made available on the Internet. One such web-based tool has been designed to help with national planning regarding active case finding, with mathematical models to help assess the likely cost of different strategies per case detected when applied to different populations. ${ }^{56}$

Web-based tools can also be used to monitor service provision and quality across wide geographic areas. They allow auditing of interventions, comparison between services, and the formation of large datasets for indepth epidemiologic analysis. With access to the Internet, such databases and facilities can be shared remotely over long distances and information can be uploaded in real-time, for example the use of a web-based system to study TB epidemiology in Peru. ${ }^{57}$ In the US, all TB surveillance data are held in the web-based National TB Surveillance System. The National TB Indicators Project is a secure web-based monitoring system that uses surveillance data from the National TB Surveillance System to measure the performance of local TB programs and track progress towards national TB program objectives. ${ }^{58}$ Manangan et $\mathrm{al}^{59}$ reported the use of this system to develop quality assurance strategies, through monitoring usage of the National TB Surveillance System before and after targeted training in quality assurance. In another US study, a webbased program was used to monitor staff adherence with infection control measures, one of which was tuberculin skin testing to reduce nosocomial transmission of TB. The web-based system facilitated data collection and analysis, allowing the development of more effective educational interventions to improve practice. ${ }^{60}$ Finally, Wang et al ${ }^{61}$ 
describe how the development of a national web-based TB reporting system improved compliance with national policy on TB treatment.

\section{Teaching and training}

Web-based educational resources provide new opportunities for teaching and training in TB at a local level and also through learning networks such as the National TB Curriculum Consortium in the US. ${ }^{62}$ One study reviewed teaching on TB at Brazilian nursing schools and found that, although most teaching occurred in a traditional classroom setting, teachers regularly used the Internet to update their knowledge prior to classes. ${ }^{63} \mathrm{~A}$ major advantage of web-based over traditional learning materials is the potential to provide an interactive forum with instant feedback. ${ }^{64} \mathrm{Walsh}^{65}$ described an online training package incorporating pre and post tests, interactive case histories, and up to date information, and demonstrated that learners significantly increased their knowledge and found the tool useful. A web-based format may also be used for simulation training, which can provide a more valuable educational experience than the traditional classroom setting. ${ }^{66}$ Use of telemedicine, teleradiology, and decision support systems, as described earlier, can provide valuable training to clinicians managing TB patients, as well as contribute to patient care.

The Internet is a source of vast amounts of data on TB. General information on TB can be accessed through the websites of national and WHO TB programs, and organizations such as the International Union against Tuberculosis and Lung Disease and the Stop TB Partnership. Health care professionals can access epidemiologic data from across the globe through the WHO website, as well as specific guidance on aspects of TB. Information is also available to health care professionals through online health "libraries" such as the national electronic library for health in the UK. ${ }^{67}$ More focused specialist information is also available online, for example, the BCG world "atlas" which provides information on BCG vaccination practices in many countries to aid clinical management of patients. ${ }^{68}$ Several early publications provide useful summaries of general TB resources on the Internet, ${ }^{69,70}$ but to our knowledge have not been updated more recently.

\section{Conclusion}

In this review, we have explored scenarios in the diagnosis, treatment, and surveillance of TB where web-based technologies are now contributing to improved service provision and TB control. We have touched on the role of new technologies in designing and monitoring national TB control programs, and in facilitating the delivery of teaching and training. There is no doubt that web-based technologies will be used increasingly globally in the fight against TB, despite the practical and financial challenges associated with their use in remote or resource-poor settings, and the development of suitable online systems and infrastructure at a national level needs to be prioritized to ensure that the potential of these new developments can be maximized.

\section{Disclosure}

The authors report no conflicts of interest in this work.

\section{References}

1. World Health Organisation Global Tuberculosis report, 2012. Available from: http://www.who.int/tb/publications/global_report/en/index.html. Accessed July 30, 2013.

2. Raviglione MC. The Global Plan to Stop TB, 2006-2015. Int J Tuberc Lung Dis. 2006;10:238-239.

3. Blaya JA, Fraser HS, Holt B. E-health technologies show promise in developing countries. Health Aff. 2010;29:244-251.

4. Simba DO. Application of ICT in strengthening health information systems in developing countries in the wake of globalisation. Afr Health Sci. 2004;4:194-199.

5. Steingart KR, Sohn H, Schiller I, et al. Xpert(R) MTB/RIF assay for pulmonary tuberculosis and rifampicin resistance in adults. Cochrane Database Syst Rev. 2013;1:CD009593.

6. Feng Y, Liu S, Wang Q, et al. Rapid diagnosis of drug resistance to fluoroquinolones, amikacin, capreomycin, kanamycin and ethambutol using genotype MTBDRsl assay: a meta-analysis. PLoS One. 2013;8: e55292.

7. Paglia MG, Bevilacqua N, Haji HS, et al. Improvement of tuberculosis laboratory capacity on Pemba Island, Zanzibar: a health cooperation project. PLoS One. 2012; 7:e44109.

8. Shin SS, Yagui M, Ascencios L, et al. Scale-up of multidrug-resistant tuberculosis laboratory services, Peru. Emerg Infect Dis. 2008;14: 701-708.

9. Gandhi NR, Nunn P, Dheda K, et al. Multidrug-resistant and extensively drug-resistant tuberculosis: a threat to global control of tuberculosis. Lancet. 2010;375:1830-1843.

10. Coulborn RM, Panunzi I, Spijker S, et al. Feasibility of using teleradiology to improve tuberculosis screening and case management in a district hospital in Malawi. Bull World Health Organ. 2012;90:705-711.

11. Corr P. Teleradiology in KwaZulu-Natal. A pilot project. S Afr Med J. 1998;88:48-49.

12. Jarvis L, Stanberry B. Teleradiology: threat or opportunity. Clin Radiol. 2005;60:840-845.

13. The Royal College of Radiologists. Standards for the provision of teleradiology within the United Kingdom. London, UK: The Royal College of Radiologists, 2010. Available from: http://www.rcr. ac.uk/docs/radiology/pdf/bfcr(10)7_stand_telerad.pdf. Accessed July 30, 2013.

14. Dunbar R, Lawrence K, Verver S, et al. Accuracy and completeness of recording of confirmed tuberculosis in two South African communities. Int J Tuberc Lung Dis. 2011;15:337-443.

15. Blaya JA, Shin SS, Yale G, et al. Electronic laboratory system reduces errors in National Tuberculosis Program: a cluster randomized controlled trial. Int J Tuberc Lung Dis. 2010;14:1009-1015.

16. Blaya JA, Cohen T, Rodriguez P, Kim J, Fraser HS. Personal digital assistants to collect tuberculosis bacteriology data in Peru reduce delays, errors, and workload, and are acceptable to users: cluster randomized controlled trial. Int J Infect Dis. 2009;13:410-418. 
17. Blaya JA, Shin S, Contreras C, et al. Full impact of laboratory information system requires direct use by clinical staff: cluster randomized controlled trial. J Am Med Inform Assoc. 2011;18:11-16.

18. Chin CD, Cheung YK, Laksanasopin T, et al. Mobile device for disease diagnosis and data tracking in resource-limited settings. Clin Chem. 2013;59:629-640.

19. Menzies D, Gardiner G, Farhat M, Greenaway C, Pai M. Thinking in three dimensions: a web-based algorithm to aid the interpretation of tuberculin skin test results. Int J Tuberc Lung Dis. 2008;12:498-505.

20. Steele AW, Eisert S, Davidson A, et al. Using computerised clinical decision support for latent tuberculosis screening. Am J Prev Med. 2005;28:281-284

21. Uldal SB, Nikishova E, Rakova N, Mariandyshev A, Bye SM, Nisovtszeva N. Using e-mail in the management of tuberculosis patients, north-west Russia. Int J Tuberc Lung Dis. 2005;9:1367-1372.

22. Zolfo M, Lynen L, Dierckx J, Colebunders R. Remote consultations and HIV/AIDS continuing education in low-resource settings. Int $J$ Med Inform. 2006;75:633-637.

23. Royal Society of Medicine. Wootton R, Patil NG, Scott RE, Ho K, editors. Telehealth in the Developing World. London, UK: Royal Society of Medicine Press; 2009.

24. Lau F, Price M, Boyd J, Partridge C, Bell H, Raworth R. Impact of electronic medical record on physician practice in office settings: a systematic review. BMC Med Inform Decis Mak. 2012;12:10.

25. Fraser HS, Biondich P, Moodley D, Choi S, Mamlin BW, Szolovits P. Implementing electronic medical record systems in developing countries. Inform Prim Care. 2005;13:83-95.

26. Fraser H, Choi SS, Galipot M, Jazayeri D, Mangubat N. Successful transfer of a web-based TB medical record from Peru to the Philippines. AMIA Annu Symp Proc. 2006:924.

27. Open Medical Record System. Available from: http://openmrs.org. Accessed July 30, 2013.

28. Furuie SS, Rebelo MS, Moreno RA, et al. Managing medical images and clinical information: InCor's experience. IEEE Trans Inf Technol Biomed. 2007;11:17-24.

29. Ballard DW, Rauchwerger AS, Reed ME, et al. Emergency physicians' knowledge and attitudes of clinical decision support in the electronic health record: a survey-based study. Acad Emerg Med. 2013;20: $352-360$.

30. Garrett NY, Yasnoff WA. Disseminating public health practice guidelines in electronic medical record systems. J Public Health Manag Pract. 2002;8:1-10.

31. Moulding TS. Medication monitors to treat tuberculosis. A supplement to directly observed therapy. Am J Resp Crit Care Med. 1999;159: 989-991.

32. Amet I, Walter PN, Hersberger KE. Polymedication electronic monitoring system (POEMS) - a new technology for measuring adherence. Front Pharmacol. 2013;4:1-6.

33. Krueger K, Ruby D, Cooley P, et al. Videophone utilization as an alternative to directly observed therapy for tuberculosis. Int J Tuberc Lung Dis. 2010;14:779-781.

34. Fraser HS, Allen C, Bailey C, Douglas G, Shin S, Blaya J. Information systems for patient follow-up and chronic management of HIV and tuberculosis: a life-saving technology in resource-poor areas. $J$ Med Internet Res. 2007;9:e29.

35. Fraser HS, Blaya J, Choi SS, Bonilla C, Jazayeri D. Evaluating the impact and costs of deploying an electronic medical record system to support TB treatment in Peru. AMIA Annu Symp Proc. 2006:264-268.

36. Singla D, Anurag M, Dash D, Raghava GP. A web server for predicting inhibitors against bacterial target GlmU protein. BMC Pharmacol. 2011;11:5

37. Chanumolu SK, Rout C, Chauhan RS. UniDrug-target: a computational tool to identify unique drug targets in pathogenic bacteria. PLoS One. 2012;7:e32833.

38. Raftery J. Target selection: triage in the structural genomics battlefield. Methods Mol Biol. 2008;426:37-47.
39. McMurry J, Sbai H, Gennaro ML, Carter EJ, Martin W, de Groot AS. Analyzing Mycobacterium tuberculosis proteomes for candidate vaccine epitopes. Tuberculosis (Edinb). 2005;85:95-105.

40. Hassan S, Logambiga P, Raman AM, Subazini TK, Kumaraswami V, Hanna LE. MtbSD - a comprehensive structural database for Mycobacterium tuberculosis. Tuberculosis (Edinb). 2011;91:556-562.

41. Ehlman DC, Jackson M, Saenz G, et al. Evaluation of an innovative Internet-based partner notification program for early syphilis case management, Washington, DC, January 2007-June 2008. Sex Transm Dis. 2010;37:478-485.

42. Jenkins WD, Wold B. Use of the Internet for the surveillance and prevention of sexually transmitted diseases. Microbes Infect. 2012;14: 427-437.

43. Calderwood MS, Platt R, Hou X, et al. Real-time surveillance for tuberculosis using electronic health record data from an ambulatory practice in eastern Massachusetts. Public Health Rep. 2010;125:843-850.

44. Hanson RL, Gannon MJ, Khamo N, Sodhi M, Orr AM, Stubbings J. Improvement in safety monitoring of biologic response modifiers after the implementation of clinical care guidelines by a specialty. J Manag Care Pharm. 2013;19:49-67.

45. Crawford JT. Genotyping in contact investigations: a CDC perspective. Int J Tuberc Lung Dis. 2003;7:S453-S457.

46. Tardin A, Dominice Dao M, Janssens JP. Tuberculosis cluster in an immigrant community: case identification issues and a transcultural perspective. Trop Med Int Health. 2009;14:995-1002.

47. Weniger T, Krawczyk J, Supply P, Harmsen D, Niemann S. Online tools for polyphasic analysis of Mycobacterium tuberculosis complex genotyping data: now and next. Infect Genet Evol. 2012;12:748-754.

48. Shabbeer A, Ozcaglar C, Yener B, Bennett KP. Web tools for molecular epidemiology of tuberculosis. Infect Genet Evol. 2012;12:767-782.

49. Vishnoi A, Srivastava A, Roy R, Bhattacharya A. MGDD: Mycobacterium tuberculosis genome divergence database. $B M C$ Genomics. 2008;9:373

50. Ohkado A, Murase Y, Mori M, et al. Transmission of specific genotype streptomycin resistant strains of Mycobacterium tuberculosis in the Tokyo metropolitan area in Japan. BMC Infect Dis. 2009;9:138.

51. Lew WJ, Lee EG, Bai JY, et al. An Internet-based surveillance system for tuberculosis in Korea. Int J Tuberc Lung Dis. 2006;10: 1241-247.

52. Dominkovics P, Granell C, Perez-Navarro A, Casals M, Orcau A, Cayla JA. Development of spatial density maps based on geoprocessing web services: application to tuberculosis incidence in Barcelona, Spain. Int J Health Geogr. 2011;10:62.

53. Mor Z, Migliori GB, Althomsons SP, Loddenkemper R, Trnka L, Iademarco MF. Comparison of tuberculosis surveillance systems in lowincidence industrialised countries. Eur Respir J. 2008;32:1616-1624.

54. Vranken R, Coulombier D, Kenyon T, et al. Use of a computerised tuberculosis register for automated generation of case finding, sputum conversion and treatment outcome reports. Int J Tuberc Lung Dis. 2002;6:111-120.

55. Nadol P, Stinson KW, Coggin W, et al. Electronic tuberculosis surveillance systems: a tool for managing today's TB programs. Int J Tuberc Lung Dis. 2008;12(3 Suppl 1):8-16.

56. Nishikiori N, Van Weezenbeek C. Target prioritization and strategy selection for active case-finding of pulmonary tuberculosis: a tool to support country-level project planning. BMC Public Health. 2013;13:97.

57. Fraser HS, Thomas D, Tomaylla J, et al. Adaptation of a web-based, open source electronic medical record system platform to support a large study of tuberculosis epidemiology. BMC Med Inform Decis Mak. 2012;12:125.

58. Centers for Disease Control and Prevention. Monitoring tuberculosis programs - National Tuberculosis Indicator Project, United States, 2002-2008. MMWR Morb Mortal Wkly Rep. 2010;59:295-298.

59. Manangan LP, Tryon C, Magee E, Miramontes R. Innovative qualityassurance strategies for tuberculosis surveillance in the United States. Tuberc Res Treat. 2012;2012:481230. 
60. Ribner BS, Hall C, Steinberg JP, et al. A web-based programme to ensure compliance of medical staff providers with mandated health care facility requirements. Am J Infect Control. 2011;39: 511-514.

61. Wang L, Liu X, Huang F, Hennig C, Uplekar M, Jiang S. Engaging hospitals to meet tuberculosis control targets in China: using the Internet as a tool to put policy into practice. Bull World Health Organ. 2010;88:937-942.

62. Harrity S, Jackson M, Hoffman H, Catanzaro A. The National Tuberculosis Curriculum Consortium: a model of multi-disciplinary educational collaboration. Int J Tuberc Lung Dis. 2007;1:270-274.

63. Villa TC, Ruffino-Netto A, Andrade RL, Alarcon EA, Montero CV, Firmino DR. Survey on tuberculosis teaching in Brazilian nursing schools. Int J Tuberc Lung Dis. 2006;10:323-327.

64. Conrad PA, Hird D, Arzt J, et al. Interactive computerised learning program exposes veterinary students to challenging international animal-health problems. J Vet Med Educ. 2007;34:497-501.
65. Walsh K. Online educational tools to improve the knowledge of primary care professionals in infectious diseases. Educ Health (Abingdon). 2008;21:64.

66. Spinello EF, Fischbach R. Using a web-based simulation as a problembased learning experience: perceived and actual performance of undergraduate public health students. Public Health Res. 2008;123 Suppl 2: 78-84.

67. Roy A, Kostkova P, Catchpole M, Carson E. Web-based provision of information on infectious diseases: a systems study. Health Informatics J. 2006;12:274-292.

68. Zwerling A, Behr MA, Verma A, Brewer TF, Menzies D, Pai M. The BCG World Atlas: a database of global BCG vaccination policies and practices. PLoS Med. 2011;8:e1001012.

69. Abu-Amero K, Tuberculosis information on the web. J R Soc Promot Health. 2002;122:82-85.

70. Kato-Maeda M, Small PM. User's guide to tuberculosis resources on the Internet. Clin Infect Dis. 2001;32:1580-1588.
Clinical Epidemiology

\section{Publish your work in this journal}

Clinical Epidemiology is an international, peer-reviewed, open access journal focusing on disease and drug epidemiology, identification of risk factors and screening procedures to develop optimal preventative initiatives and programs. Specific topics include: diagnosis, prognosis, treatment, screening, prevention, risk factor modification, systematic

Submit your manuscript here: http://www.dovepress.com/clinical-epidemiology-journa

\section{Dovepress}

reviews, risk \& safety of medical interventions, epidemiology \& biostatical methods, evaluation of guidelines, translational medicine, health policies \& economic evaluations. The manuscript management system is completely online and includes a very quick and fair peer-review system, which is all easy to use. 